\title{
DADOS GOVERNAMENTAIS ABERTOS EM APLICATIVOS BRASILEIROS
}

\author{
OPEN GOVERNMENT DATA IN BRASILIAN \\ APPLICATIONS
}

\author{
Patrícia Nascimento Silvaa \\ Marta Macedo Kerr Pinheirob
}

\begin{abstract}
RESUMO
Introdução: Os dados públicos disponibilizados pelos governos através da internet são identificados como Dados Governamentais Abertos (DGA). Pela disponibilização dos dados, a pesquisa busca avaliar se os mesmos são reutilizados pela sociedade e através dos aplicativos desenvolvidos pelo governo brasileiro em produtos e serviços de informação. Objetivo: Identificar os aplicativos disponibilizados pelo governo brasileiro e seu grau de reúso baseado na utilização dos DGA. Metodologia: Através de uma pesquisa exploratória com abordagem qualitativa e quantitativa foram analisados os aplicativos disponibilizados no Guia de Aplicativos do Governo Federal e avaliados critérios de acesso, as fontes de dados e principalmente se utilizavam DGA. Resultados: Foram identificados 30 aplicativos que utilizam DGA, um número expressivo de reúso quando comparado a quantidade de aplicativos oficiais produzidos com DGA do governo brasileiro. Porém ainda há uma quantidade significativa de jogos e aplicativos que não utilizam DGA. Conclusões: Há uma evolução no uso dos DGA brasileiros, mas em relação ao imenso potencial dos DGA, - Brasil ainda caminha inexpressivamente no que diz respeito ao uso dos DGA em produtos e serviços de informação.
\end{abstract}

Descritores: Dados Governamentais Abertos. Informação governamental. Políticas de informação.

\footnotetext{
a Doutora em Gestão e Organização do Conhecimento pela Universidade Federal de Minas Gerais (UFMG). Analista de Tecnologia da Informação no Centro de Computação da Universidade Federal de Minas Gerais (UFMG). E-mail: patricia.inf@gmail.com

b Doutora em Ciência da Informação pelo (IBICT-UFRJ). Professora da Universidade FUMEC E-mail: martakerr@gmail.com
} 


\section{INTRODUÇÃO}

O tema Dados Governamentais Abertos (DGA) é usado recentemente para se referir aos dados públicos disponibilizado pelos governos através da internet. Ao disponibilizar estes dados espera-se que os mesmos sejam reutilizados. Essa reutilização dos dados gera um impacto maior sobre a capacidade dos cidadãos em fiscalizar os governos, cobrar eficácia e eficiência dos mesmos, além de permitir que empresas públicas e privadas utilizem os DGA para desenvolver novos serviços e produtos, estimular a inovação e a melhoria da qualidade na prestação dos serviços públicos (VAN DEN BROEK; RIJKEN; VAN OORT, 2012).

O desenvolvimento dos DGA depende de: uma política clara da Administração Pública em relação ao acesso à informação pública; modificação na estratégia para a transformação das relações entre o Estado e a sociedade e, finalmente, uma revisão do lócus central da organização pública "como intérprete das necessidades sociais e produtora direta dos serviços" (AGUNE; GREGORIO FILHO; BOLLIGER, 2010).

No Brasil a Lei de Acesso à Informação, que entrou em vigor em maio de 2012, é citada na academia como o arcabouço jurídico para a obrigação governamental de disponibilizar dados abertos no país (ISOTANI; BITTENCOURT, 2015). Mas a simples disponibilidade dos DGA torna-se uma medida "incompleta" que demanda outras ações para sua avaliação. Uma política de informação pública precisa ir além de um foco sobre os dados ao desenvolver formas de examinar, desafiar, re-imaginar e re-calibrar as prioridades, os raciocínios e métodos de infraestruturas de informação pública, holisticamente concebidas. Além disso, é preciso um olhar através das informações divulgadas, com a preocupação em mensurar e apresentar uma concepção mais clara da utilização desses dados, e das possíveis infraestruturas e suas formas de operar no mundo (GRAY, 2015).

A disponibilização de dados abertos gera benefícios para a gestão interna das organizações por ampliar o potencial de melhoria dos processos, apresentar um retorno à sociedade e minimizar a cultura de resguardar dados 
que não comprometem a segurança. Dutra e Lopes (2013) demonstram que apesar do governo usar dados abertos de órgãos de uma mesma esfera ou de setores distintos, em maior integração de dados e de sistemas dentro das estruturas de governo, o uso ainda é incipiente.

Na prática, os DGA são utilizados de forma indireta pela sociedade através de aplicativos. Estes aplicativos são desenvolvidos pela sociedade (através de programadores de computador que possuem competência técnica para tal) e, também, para a sociedade (público e cidadão final), com o objetivo de possibilitar a análise e processamento dos dados (MOREIRA, 2015).

O Portal Brasileiro de Dados Abertos é o portal oficial do governo brasileiro para a disponibilização de DGA. Juntamente com este portal foi criada a sessão de 'Aplicativos' desenvolvidos pela sociedade civil, assim listados: Reputação S.A., Reclamações Procon, Reclamações BR, Para onde foi o meu dinheiro, Onde acontece, Aeroportos Brasil, Siga seu vereador, Radar Parlamentar, Fala, Câmara, Basômetro e Painel do PAC, produzidos por organizações públicas e pela sociedade civil. Os aplicativos que foram criados por organizações públicas podem ser visualizados no portal Vispublica: http://vispublica.gov.br/vispublica/, que apresenta modelos de visualização de dados públicos, e os aplicativos oficiais, que não necessariamente utilizam dados abertos, podem ser encontrados no Guia de Aplicativos do Governo Federal: http://aplicativos.gov.br (BRASIL, 2016).

O guia é conceituado como catálogo de aplicativos para dispositivos móveis criados por órgãos do poder executivo federal. Funciona como um portal centralizador, no qual o cidadão obtém informações, de forma gratuita, de responsabilidade governamental (BRASIL, 2016). Os produtos e serviços produzidos pelos órgãos públicos podem utilizar os próprios DGA disponibilizados pelo próprio órgão e ainda através do cruzamento com dados de outras fontes criar novos aplicativos que otimizem serviços disponibilizáveis para os cidadãos. Esta pesquisa ${ }^{1}$ tem o objetivo de analisar as formas de

\footnotetext{
${ }^{1}$ Este artigo é resultado parcial da tese de doutorado intitulada Dados Governamentais Abertos: métricas e indicadores de reúso, defendida em março de 2018, no Programa de PósGraduação em Gestão e Organização do Conhecimento da Universidade Federal de Minas Gerais por Patrícia Nascimento Silva, orientado por Marta Macedo Kerr Pinheiro.
} 
acesso e aspectos relacionados ao uso e aplicação dos DGA brasileiros no Guia de Aplicativos do Governo Federal.

\section{DADOS GOVERNAMENTAIS ABERTOS}

Open Government Data ou DGA é conceituado por diferentes áreas e autores. Agune, Gregorio Filho e Bolliger (2010) definem como a "[...] disponibilização, através da Internet, de informações e dados governamentais de domínio público para a livre utilização pela sociedade." Após uma pesquisa na literatura sobre o conceito de DGA observou-se que o termo também é traduzido como Dados Abertos Governamentais por alguns autores e instituições, porém nesta pesquisa será considerado o termo DGA. O Quadro 1 apresenta algumas definições e seus respectivos autores:

\section{Quadro 1 - Definições de DGA na literatura}

\begin{tabular}{|c|c|}
\hline Autor & Definição \\
\hline Barbalho (2014) & $\begin{array}{l}\text { [...] "o termo "dados abertos governamentais" refere-se aos elementos } \\
\text { gerados nos processos de governo que passam a ser disponibilizados } \\
\text { para consumo de outros atores". }\end{array}$ \\
\hline $\begin{array}{c}\text { Albano e Araújo } \\
(2013)\end{array}$ & $\begin{array}{l}\text { Dados Governamentais Abertos é uma plataforma tecnológica adotada } \\
\text { pelos governos para atender as demandas da nova sociedade. Este tema } \\
\text { é baseado nos pressupostos de transparência, colaboração e } \\
\text { participação. Acredita-se que com essas iniciativas, é possível gerar } \\
\text { atividades econômicas e sociais de valor através do uso de dados } \\
\text { públicos pela sociedade. }\end{array}$ \\
\hline $\begin{array}{l}\text { Kalampokis, } \\
\text { Tambouris e } \\
\text { Tarabanis (2011) }\end{array}$ & $\begin{array}{l}\text { Dados Governamentais Abertos refere-se ao setor público, tornando a } \\
\text { informação livremente disponível em formatos abertos e permitindo vias } \\
\text { de acesso públicas que facilitem a exploração. }\end{array}$ \\
\hline $\begin{array}{c}\text { Yannoukakou e } \\
\text { Araka (2014) }\end{array}$ & $\begin{array}{l}\text { Dados Governamentais Abertos refere-se a dados produzidos ou } \\
\text { encomendados pelo governo ou entidades controladas pelo governo, que } \\
\text { pode ser usado livremente, reutilizados e redistribuído por qualquer } \\
\text { pessoa. }\end{array}$ \\
\hline Solar et al. (2014) & $\begin{array}{l}\text { O conceito de DGA é uma filosofia de trabalho para capacitar os cidadãos } \\
\text { e proporcionar-lhes acesso e licença para usar os dados gerados por } \\
\text { entidades públicas, de modo que eles podem usar, armazenar, redistribuir } \\
\text { e integrá-los com outras fontes de dados. }\end{array}$ \\
\hline W3C (2016) & $\begin{array}{l}\text { Dados Abertos Governamentais são a publicação e disseminação das } \\
\text { informações do setor público na Web, compartilhadas em formato bruto e } \\
\text { aberto, compreensíveis logicamente, de modo a permitir a sua reutilização } \\
\text { em aplicações digitais desenvolvidas pela sociedade. }\end{array}$ \\
\hline
\end{tabular}
Fonte: Elaborado pelos autores.

Os conceitos apresentados no Quadro 1 demonstram um mesmo direcionamento para o conceito de DGA e indicam tratar-se de dados públicos disponibilizados pelos governos e que podem ser livremente reutilizados pela 
sociedade. É reconhecida a importância dos governos como grandes produtores e colecionadores de dados em diferentes domínios. Dados geoespaciais, ambientais, de transporte, planejamento ou orçamento tem valor social e comercial podendo ser usados com propósitos diferentes dos que foram originalmente previstos (ALEXOPOULOS et al., 2014). Além disso, a disponibilização desses dados incentiva o governo e partes interessadas a inovar e criar novos serviços. A criação de um novo produto de informação é exemplificado na Figura 1 em meio a um grande conjunto de dados:

Figura 1 - Como os dados abertos podem gerar outros conjuntos de dados

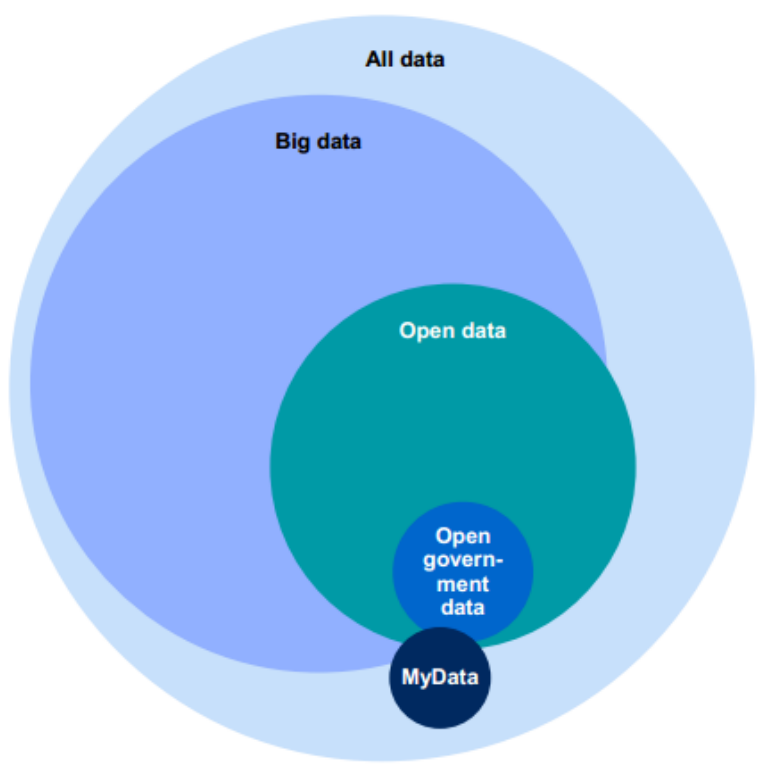

Fonte: McKinsey Global Institute (2013, p. 4).

Em um contexto que envolve todos os dados disponibilizados na web, All data, os diferentes tipos de dados formam subconjuntos com diversas nomenclaturas como Big data, que envolve diversos conjuntos de dados, o Open data, que corresponde somente aos dados abertos e o Open government data que corresponde aos dados governamentais abertos. A representação MyData demonstra que todos estes tipos de dados podem ser utilizados para criar uma nova aplicação.

Além de considerar os conceitos, os princípios dos DGA também conhecidos como "8 Open Government Data Principles" ou "Sebastopol Principles", definidos em 2007 por especialistas, em Sebastopol, Califórnia, Estados Unidos, foram considerados uma forma de validação para identificar 
os DGA (OPEN GOV DATA, 2007). Conforme W3C (2016) os oito princípios definem que:

1. Os dados devem estar completos: todos os dados devem estar disponíveis;

2. Os dados devem ser primários: os dados são apresentados tais como coletados na fonte, com o maior nível possível de granularidade e sem agregação ou modificação;

3. Os dados devem ser atualizados: os dados devem ser disponibilizados tão rapidamente quanto necessário à preservação do seu valor;

4. Os dados devem estar acessíveis: os dados devem ser disponibilizados para o maior alcance possível de usuários e para o maior conjunto possível de finalidades;

5. Os dados devem ser processados por máquina: os dados devem estar razoavelmente estruturados de modo a possibilitar processamento automatizado;

6. O acesso deve ser não discriminatório: os dados devem estar disponíveis para todos, sem exigência de requerimento ou cadastro;

7. Os formatos de dados devem ser não proprietários: os dados devem estar disponíveis em formato sobre o qual nenhuma entidade detenha controle exclusivo;

8. Os dados devem ser livres de licença: os dados não estão sujeitos a nenhuma restrição de direito autoral, patente, propriedade intelectual ou segredo industrial. Restrições sensatas à privacidade, segurança e privilégios de acesso são permitidas.

Desta forma, para identificar aplicativos que utilizam DGA devem ser observados todos os conceitos relativos aos DGA e os seus princípios.

\section{REFERENCIAL METODOLÓGICO}

A metodologia consistiu em uma pesquisa exploratória com abordagem qualitativa e quantitativa e como campo de aplicação o Guia de Aplicativos do 
Governo Federal. A pesquisa foi realizada entre agosto e dezembro de 2016 e foram identificados 129 aplicativos desenvolvidos por órgãos públicos.

Para identificar os aplicativos que realmente usam os conceitos e os princípios dos DGA foi feita a instalação de todos os aplicativos. Por se tratar de uma grande quantidade de aplicativos para dispositivos móveis, que podem apresentar limitações no processamento e armazenamento desta quantidade de aplicativos, optou-se por simular o sistema operacional em uma máquina virtual.

O sistema operacional Android foi utilizado no ambiente de teste dos aplicativos por ser a plataforma que contemplava mais de $90 \%$ dos aplicativos disponibilizados e por ser distribuído gratuitamente. Além disso, o Android domina o mercado mundial de smartphones e é o sistema operacional mais utilizado no Brasil (IDC, 2016; IDC Brasil, 2016).

Após realizar testes para verificar o desempenho e a usabilidade do Android em máquinas virtuais, instalações limpas em desktops e alguns softwares emuladores para Android foi selecionado o software MEMU App Player (http://www.memuplay.com/), um software gratuito que emula o sistema operacional Android. Como o Portal Brasileiro de Dados Abertos foi criado em 2012, para disponibilizar conjuntos de DGA, optou-se por utilizar a versão 4.2.2, uma versão intermediária, que é compatível com os aplicativos mais antigos (2012) e com os mais recentes (2016).

Para classificar os aplicativos quanto à forma de acesso, os mesmos foram instalados e analisadas as exigências para seu uso. Assim, quando não fossem solicitados cadastros ou somente dados comuns a qualquer cidadão, 0 aplicativo seria considerado como aberto e quando fossem solicitados cadastros de qualquer natureza ou a exigência de dados privados, específicos da aplicação ou que restringisse parte da população, o aplicativo seria considerado como restrito. Apesar de alguns aplicativos demandarem um cadastro simples ou utilizarem logins já existentes em redes sociais, estes foram classificados como restrito, pois considerou-se a demanda de qualquer forma de identificação, estando em não conformidade com os princípios dos DGA. Destaca-se que não foram analisadas as permissões de acesso 
atribuídas na instalação dos aplicativos, uma vez que estes dados geralmente são utilizados para estatísticas dos desenvolvedores e fogem ao foco desta pesquisa.

Todos os aplicativos com acesso aberto, disponíveis para a plataforma Android e que não apresentaram erros na instalação ou em seu uso foram analisados. Para identificar as categorias de DGA utilizados em cada aplicativo foram assumidas as categorias de classificação, já utilizadas no Guia, juntamente com a análise do tipo de informação disponibilizada baseado nos testes executados nos aplicativos.

\section{RESULTADOS E DISCUSSÃO}

Foram analisados 129 aplicativos de acordo com as categorias definidas pelo Guia e as informações disponíveis, sendo atribuída uma classificação pela forma de acesso (Quadro 2).

Quadro 2 - Aplicativos disponibilizados no Guia de Aplicativos do Governo Federal

\begin{tabular}{|l|l|c|}
\hline \multicolumn{1}{|c|}{ Aplicativo } & \multicolumn{1}{c|}{ Categoria } & Tipo de Acesso \\
\hline PESSOA FÍSICA & Economia e emprego & Aberto \\
\hline BANCO DO BRASIL & Economia e emprego & Restrito \\
\hline VIAJANTES NO EXTERIOR & Economia e emprego & Aberto \\
\hline CAFÉS DO BRASIL & Economia e emprego & Indisponível \\
\hline CAIXA & Economia e emprego & Restrito \\
\hline SEBRAE & Economia e emprego & Aberto \\
\hline AUTOATENDIMENTO SETOR PÚBLICO & Economia e emprego & Restrito \\
\hline LOTERIAS CAIXA & Economia e emprego & Aberto \\
\hline CAIXA PARA TABLETS & Economia e emprego & Aberto \\
\hline FGTS NA MINHA VIDA & Economia e emprego & Aberto \\
\hline IMPORTADOR & Economia e emprego & Aberto \\
\hline CALCULADORA DO CIDADÃO & Economia e emprego & Aberto \\
\hline DINHEIRO BRASILEIRO & Economia e emprego & Incompatível \\
\hline IBGE & Economia e emprego & Aberto \\
\hline COLABONAÇÃO & Economia e emprego & Aberto \\
\hline VACINAÇÃO EM DIA & Saúde & Aberto \\
\hline ACABE COM A DENGUE & Saúde & Aberto \\
\hline STRESSLAB - CONTROLE DO STRESS & Saúde & Restrito \\
\hline MEU BOCÃO & Saúde & Aberto \\
\hline VACINAÇÃO! & Saúde & Aberto \\
\hline PCDT CRIANÇA E ADOLESCENTE & Saúde & Aberto \\
\hline PCDT ADULTO & Saúde & Aberto \\
\hline HORUS CIDADÃO & Saúde & Restrito \\
\hline CHECKMYECG & Saúde & Incompatível \\
\hline DEFESORES DO CORPO HUMANO & Saúde & Aberto \\
\hline MEU DIA ALIMENTAR & Saúde & Aberto \\
\hline PSF + & Saúde & Aberto \\
\hline BRASIL EM CIDADES & Cidadania e Justiça & Aberto \\
\hline STJ - CONSULTA PROCESSUAL & Cidadania e Justiça & Aberto \\
\hline PROTEJABRASIL & Cidadania e Justiça & \\
\hline CÂMARA NOTÍCIAS & Cidadania e Justiça & \\
\hline & & \\
\hline
\end{tabular}




\begin{tabular}{|c|c|c|}
\hline SINESP CIDADÃO & Cidadania e Justiça & Aberto \\
\hline DENUNCIE A DISCRIMINAÇÃO & Cidadania e Justiça & Aberto \\
\hline $\begin{array}{l}\text { GUIA DE GÊNERO, RAÇA E ETNIA PARA } \\
\text { JORNALISTAS }\end{array}$ & Cidadania e Justiça & Aberto \\
\hline ESTATUTO DA IGUALDADE RACIAL & Cidadania e Justiça & Aberto \\
\hline CORREIOS MOBILE & Cidadania e Justiça & Aberto \\
\hline SIMVIDA & Cidadania e Justiça & Indisponível \\
\hline PREVAPP - SIMULADOR INSS & Cidadania e Justiça & Aberto \\
\hline SIGALEI & Cidadania e Justiça & Aberto \\
\hline TRÂNSITO LEGAL & Cidadania e Justiça & Aberto \\
\hline CARCARÁ DIREITO & Cidadania e Justiça & Aberto \\
\hline VIDA POLÍTICA & Cidadania e Justiça & Restrito \\
\hline BUS BUSTERS & Cidadania e Justiça & Restrito \\
\hline TROCA TRECO & Cidadania e Justiça & Restrito \\
\hline VOLUNTÁRIO & Cidadania e Justiça & Restrito \\
\hline ATLAS DE ACESSO À JUSTIÇA & Cidadania e Justiça & Aberto \\
\hline CONSUMIDOR.GOV.BR & Cidadania e Justiça & Aberto \\
\hline INFOLEG & Cidadania e Justiça & Erro \\
\hline MÃOS NO VOLANTE & Educação & Aberto \\
\hline MOTORISTA DA PARADA & Educação & Restrito \\
\hline HAND TALK & Educação & Aberto \\
\hline TV ESCOLA & Educação & Aberto \\
\hline SALA DE PROFESSOR & Educação & Aberto \\
\hline SISU & Educação & Aberto \\
\hline DETETIVES DA HISTÓRIA & Educação & Aberto \\
\hline KOSME & Educação & Restrito \\
\hline SALA DIGITAL & Educação & Restrito \\
\hline REDESCOBRIDOR DO BRASIL & Educação & Aberto \\
\hline SUPER AGENTE & Educação & Aberto \\
\hline PLANOS E AÇÕES & Educação & Indisponível \\
\hline CHILI CRAB E AS NOTAS MUSICAIS & Educação & Aberto \\
\hline 1500 & Educação & Erro \\
\hline JANELA MÁGICA & Educação & Aberto \\
\hline VOA VIVALDO & Educação & Indisponível \\
\hline UM QUARTO & Educação & Incompatível \\
\hline DENTAL GAMES & Educação & Aberto \\
\hline SMART STUDY PLAN & Educação & Aberto \\
\hline O QUE É, O QUE É? & Educação & Aberto \\
\hline PROJETO ABECEDÁRIO & Educação & Erro \\
\hline KRIATIVAR & Educação & Restrito \\
\hline ENEM - GO / ENEM GAME & Educação & Incompatível \\
\hline ENAPP & Educação & Aberto \\
\hline AVALIAIFS & Educação & Aberto \\
\hline BRASIL $360^{\circ}$ & Turismo & Restrito \\
\hline INFRAERO VOOS ONLINE & Turismo & Aberto \\
\hline CÂMBIO LEGAL & Turismo & Aberto \\
\hline BRASIL QUEST & Turismo & Aberto \\
\hline BRASIL CENTRAL TUR & Turismo & Indisponível \\
\hline INFRAERO AEROPERTO & Turismo & Aberto \\
\hline BRASIL EXPERIENCE MOBILE & Turismo & Incompatível \\
\hline FELLOW TRIP & Turismo & Incompatível \\
\hline MUSEU SEM PAREDES | SÃO MIGUEL DAS MISSÕES & Turismo & Indisponível \\
\hline HOTEL BRASIL & Turismo & Aberto \\
\hline MOBGUIA & Turismo & Aberto \\
\hline SIGLA & Ciência e Tecnologia & Aberto \\
\hline SUPLEMENTA CERTO & Ciência e Tecnologia & Aberto \\
\hline CNPJ & Ciência e Tecnologia & Aberto \\
\hline CARNÊ-LEÃO & Ciência e Tecnologia & Restrito \\
\hline PANDORA & Ciência e Tecnologia & Restrito \\
\hline COOPERIA & Ciência e Tecnologia & Restrito \\
\hline MBOTS- NANORROBÔS MUSCULARES & Ciência e Tecnologia & Aberto \\
\hline ANATEL SERVIÇO MÓVEL & Ciência e Tecnologia & Aberto \\
\hline VLIBRAS & Ciência e Tecnologia & Aberto \\
\hline SOFTWARE PÚBLICO BRASILEIRO & Ciência e Tecnologia & Aberto \\
\hline ERECURSOS & Governo & Aberto \\
\hline TV NBR & Governo & Aberto \\
\hline SENADO FEDERAL & Governo & Indisponível \\
\hline DESTAQUES DO GOVERNO FEDERAL & Governo & Erro \\
\hline E-RECURSOS & Governo & Duplicado \\
\hline
\end{tabular}

Inf. Inf., Londrina, v. 24, n. 1, p. 31 - 50, jan./abr. 2019. 


\begin{tabular}{|l|l|c|}
\hline PORTAL BRASIL & Governo & Incompatível \\
\hline RASTROS DA LEI & Governo & Aberto \\
\hline ANATEL CONSUMIDOR & Governo & Aberto \\
\hline SIGEPE MOBILE & Governo & Incompatível \\
\hline ANALISABRASIL.ORG & Governo & Aberto \\
\hline DETRAN MÓVEL & Governo & Aberto \\
\hline RÁDIO MARINHA & Cultura & Indisponível \\
\hline CORREIOS CULTURAL & Cultura & Aberto \\
\hline OPERAÇÃO ABAPORU & Cultura & Aberto \\
\hline BRASIL BANDA LARGA & Infraestrutura & Indisponível \\
\hline LOCALIZADOR DE POSTOS PETROBRAS & Infraestrutura & Incompatível \\
\hline EQUIPAMENTOS PÚBLICOS & Infraestrutura & Aberto \\
\hline PAISAGISMO E JARDINAGEM & Infraestrutura & Aberto \\
\hline GOTHERE & Infraestrutura & Restrito \\
\hline SCT SUREWAY & Infraestrutura & Indisponível \\
\hline QUIZ DEFESA & Defesa e Segurança & Indisponível \\
\hline CENIPA & Defesa e Segurança & Aberto \\
\hline SIAT MOBILE - BOMBEIROS ES & Defesa e Segurança & Aberto \\
\hline TRIBUTO SCANNER & Defesa e Segurança & Aberto \\
\hline DESAPARECIDOSBRASIL.ORG & Defesa e Segurança & Aberto \\
\hline NA HORA DO APERTO & Defesa e Segurança & Indisponível \\
\hline REDE DE VIZINHOS PROTEGIDOS & Defesa e Segurança & Aberto \\
\hline HELPME - SEMPRE COM VOCÉ & Defesa e Segurança & Incompatível \\
\hline APLICATIVO DA MARINHA & Defesa e Segurança & Aberto \\
\hline BASE NAVAL DO RIO DE JANEIRO & Defesa e Segurança & Aberto \\
\hline BOLETIM AO MAR & Defesa e Segurança & Aberto \\
\hline PORTAL HIDROLÓGICO DO NORDESTE & Meio Ambiente & Aberto \\
\hline FLOS & Meio Ambiente & Aberto \\
\hline EQUILIBRA & Meio Ambiente & Aberto \\
\hline MADEIRA LEGAL & Meio Ambiente & \\
\hline
\end{tabular}

Fonte: Dados da pesquisa.

Para classificação como acesso Aberto considerou-se quando não fossem solicitados cadastros e dados pessoais ou somente dados comuns a qualquer cidadão. Para a classificação restrita considerou-se quando fossem solicitados dados privados, específicos da aplicação ou que restringisse parte da população. Assim, conforme apresentado no Quadro 2, dos 129 aplicativos iniciais que foram identificados, 103 possuíam acesso aberto e estavam disponíveis na plataforma Android.

Desses 103 aplicativos, 20 apresentaram erro na execução, incompatibilidade com as configurações definidas para o ambiente de teste, estava duplicado ou estavam indisponíveis no momento da instalação, e não puderam ser analisados. Logo, a amostra utilizada, destacada em negrito no Quadro 2, foi de 83 aplicativos.

Para cada aplicativo foi analisado se o mesmo utilizava ou não dados governamentais abertos em seu contexto. Como no Guia não são divulgados especificamente as fontes de informação ou os conjuntos de dados utilizados em cada aplicativo, foi realizada uma análise de cada aplicativo com base em uma navegação em seus recursos básicos e nas informações disponibilizadas 
no Guia de Aplicativos do Governo Federal e no Portal Brasileiro de Dados Abertos que disponibiliza conjuntos de dados do governo brasileiro.

Observada a amostra foram identificados 20 aplicativos que são jogos off-line. Estes aplicativos estão de acordo com a categoria atribuída no Guia, porém não utilizam o conceito de DGA, já que foram desenvolvidos com um conjunto de dados fixos geralmente sobre um determinado tema e não recebem atualizações ou alterações periódicas, à exceção de um único aplicativo que utiliza dados do orçamento público como fonte de dados do próprio jogo. Desta forma foram observados três grandes tipos de aplicativos: os jogos, que possuem uma temática específica e não demandam de atualizações, os aplicativos que utilizam DGA e os aplicativos que não utilizam DGA. O Gráfico 1 ilustra essa divisão:

Gráfico 1 - Definição dos tipos de aplicativos analisados

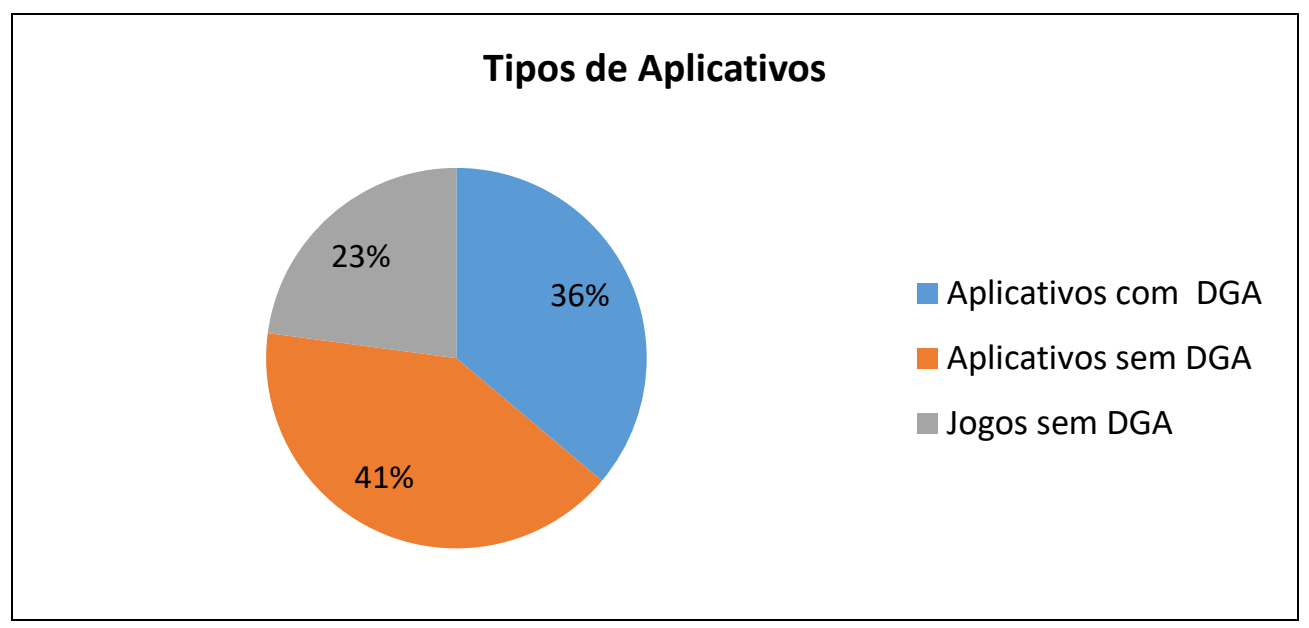

Fonte: Dados da pesquisa.

Os aplicativos que utilizam DGA, correspondente a $36 \%$ da amostra, estão distribuídos entre as categorias: Economia e emprego, Cidadania e Justiça, Educação, Turismo, Ciência e Tecnologia, Governo, Defesa e Segurança e Meio Ambiente conforme apresentado no Gráfico 2: 
Gráfico 2 - Categoria dos Aplicativos que utilizam DGA

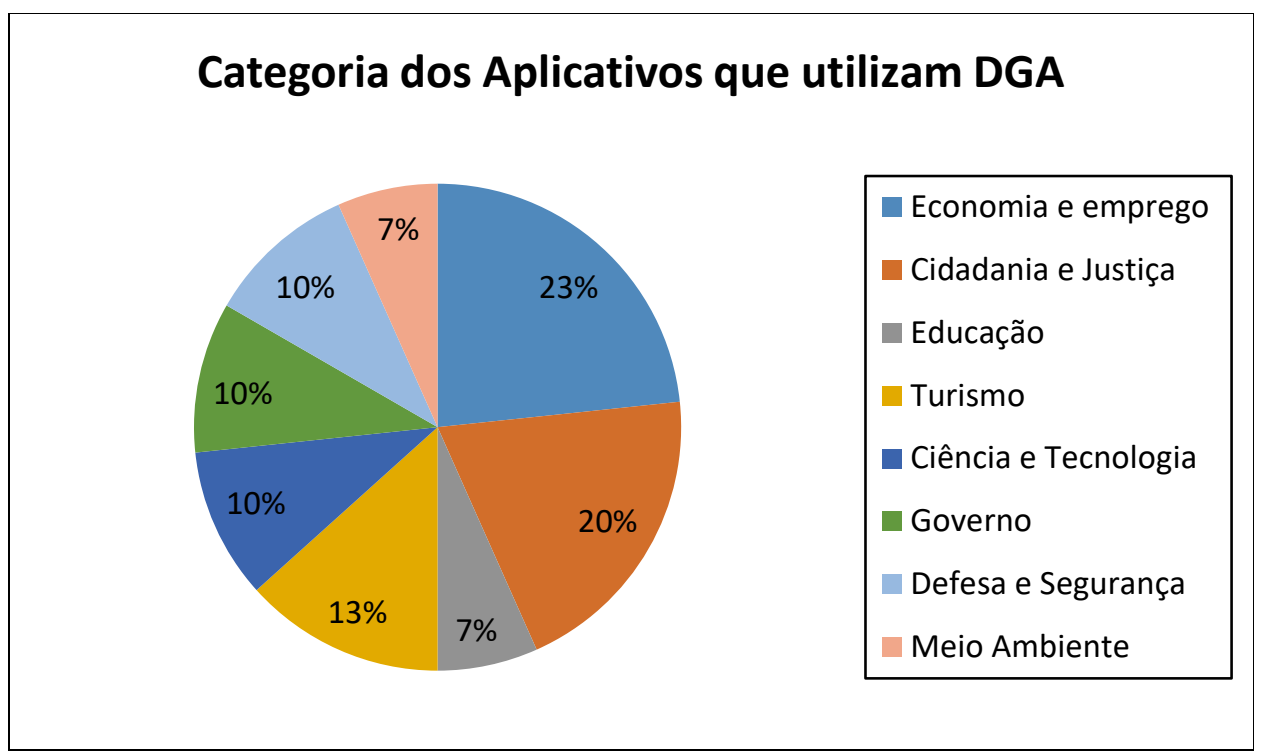

Fonte: Dados da pesquisa.

As categorias Economia e emprego e Cidadania e Justiça disponibilizaram maior número de aplicativos com o uso dos DGA, por já possuírem, antes de 2012, sistemas informatizados, com serviços como consultas a situação cadastral do CPF e a consulta processual no Superior Tribunal de Justiça (STJ). As mesmas englobam aplicativos com serviços já disponibilizados à população através dos portais, em função do atendimento a Lei de Acesso à Informação que está em vigor desde 2012.

Quando analisados os aplicativos que utilizam DGA, foram identificados os tipos de informações disponibilizadas e as possíveis bases de dados que disponibilizam estes dados, conforme as informações fornecidas pelo próprio aplicativo (Quadro 3). Porém deve-se destacar que esta correlação foi feita através do tipo de informação disponibilizada pelo aplicativo e não há dados oficiais que detalhem ou confirmem a origem das fontes de informação utilizadas. Para tanto foram considerados os conceitos de DGA, os conjuntos de dados e serviços já disponibilizados pelo Governo Federal.

Dos aplicativos que utilizam DGA, $17 \%$ se destacam pela quantidade de informações disponibilizadas e por acessar dados de mais de uma fonte de informação. O aplicativo Sinesp Cidadão, da categoria Cidadania e Justiça, por exemplo, permite realizar consultas com base no Sistema Nacional de Informações de segurança pública, prisionais e sobre drogas, foi desenvolvido 
pela SERPRO e conforme o Google play até novembro de 2016 teve mais de cinco milhões de downloads. Os aplicativos que utilizam as bases da Receita Federal e Previdência Social também permitem a consulta de dados atualizados facilitando o acesso dos cidadãos aos serviços disponibilizados pelos órgãos. É importante destacar que $90 \%$ dos aplicativos que utilizam DGA possuem informações na plataforma para download ou algum tópico de ajuda ou utilização. Esse tipo de informação é relevante, pois permite maior abrangência da população evitando a exclusão digital dos indivíduos.

Conforme os oito princípios que definem os DGA, os itens com menor adesão nesta análise são relativos à atualização e 0 acesso não discriminatório, pois só é possível recuperar a data da última versão dos aplicativos e não da atualização dos dados.

\section{Quadro 3 - Possíveis fontes dos DGA utilizados nos aplicativos}

\begin{tabular}{|c|c|c|c|}
\hline Aplicativo & Conteúdo & $\begin{array}{c}\text { Fonte } \\
\text { (Banco de dados / Conjunto } \\
\text { de dados / Portal / Serviço) }\end{array}$ & $\begin{array}{c}\text { *Última } \\
\text { atualização }\end{array}$ \\
\hline PESSOA FÍSICA & $\begin{array}{l}\text { Informações sobre CPF e restituições } \\
\text { do IRRF }\end{array}$ & Bases da Receita Federal & $24 / 02 / 2017$ \\
\hline $\begin{array}{l}\text { VIAJANTES NO } \\
\text { EXTERIOR }\end{array}$ & $\begin{array}{l}\text { Informações e consultas para } \\
\text { viajantes }\end{array}$ & Bases da Receita Federal & $01 / 02 / 2017$ \\
\hline LOTERIAS CAIXA & Informações e Resultados & $\begin{array}{l}\text { Bases da Caixa Econômica } \\
\text { Federal }\end{array}$ & $31 / 05 / 2015$ \\
\hline IMPORTADOR & $\begin{array}{l}\text { Informações e simulações sobre } \\
\text { importação }\end{array}$ & Bases da Receita Federal & $21 / 01 / 2014$ \\
\hline $\begin{array}{l}\text { CALCULADORA } \\
\text { DO CIDADÃO }\end{array}$ & $\begin{array}{l}\text { Informações e simulação de serviços } \\
\text { financeiros }\end{array}$ & Bases do Banco Central & $15 / 09 / 2016$ \\
\hline IBGE & $\begin{array}{l}\text { Informações sobre indicadores } \\
\text { econômicos, dados censitários, } \\
\text { índices de preços e notícias }\end{array}$ & Bases do IBGE & $13 / 02 / 2017$ \\
\hline COLABONAÇÃO & $\begin{array}{l}\text { Informações e simulador do } \\
\text { orçamento público }\end{array}$ & $\begin{array}{l}\text { Bases do MPOG sobre } \\
\text { Orçamento Público }\end{array}$ & $08 / 03 / 2015$ \\
\hline $\begin{array}{l}\text { BRASIL EM } \\
\text { CIDADES }\end{array}$ & $\begin{array}{l}\text { Informações sobre as cidades do } \\
\text { Brasil }\end{array}$ & $\begin{array}{l}\text { Sistema Nacional de } \\
\text { Informações das Cidades }\end{array}$ & $27 / 03 / 2013$ \\
\hline $\begin{array}{l}\text { STJ - CONSULTA } \\
\text { PROCESSUAL }\end{array}$ & Informações sobre processos do STJ & $\begin{array}{l}\text { Consulta processual do Portal } \\
\text { STJ }\end{array}$ & $25 / 02 / 2015$ \\
\hline $\begin{array}{l}\text { CÂMARA } \\
\text { NOTÍCIAS }\end{array}$ & Informações e notícias institucionais & $\begin{array}{l}\text { Portal Câmara Notícias } \\
\text { (noticias.camara.leg.br) }\end{array}$ & $08 / 05 / 2015$ \\
\hline $\begin{array}{l}\text { SINESP } \\
\text { CIDADÃO }\end{array}$ & $\begin{array}{l}\text { Informações sobre veículos roubados } \\
\text { e pessoas procuradas pela justiça }\end{array}$ & $\begin{array}{l}\text { Banco de dados do } \\
\text { Departamento Nacional de } \\
\text { Trânsito (DENATRAN) } \\
\text { Portal do CNJ } \\
\text { (http://www.cnj.jus.br) }\end{array}$ & $10 / 02 / 2017$ \\
\hline SIGALEI & Informações sobre projetos de lei & $\begin{array}{l}\text { Projetos de lei no Congresso } \\
\text { Nacional e Assembleia de } \\
\text { Minas Gerais }\end{array}$ & $04 / 04 / 2015$ \\
\hline $\begin{array}{l}\text { ATLAS DE } \\
\text { ACESSO À } \\
\text { JUSTIÇA }\end{array}$ & Informações e localização de órgãos & $\begin{array}{l}\text { Portal do acesso à Justiça } \\
\text { (acessoajustica.gov.br) }\end{array}$ & $18 / 01 / 2015$ \\
\hline SISU & $\begin{array}{l}\text { Informações sobre o Sistema de } \\
\text { Seleção Unificada (Sisu) }\end{array}$ & $\begin{array}{l}\text { Portal SISU } \\
\text { (http://sisu.mec.gov.br/) }\end{array}$ & $25 / 01 / 2017$ \\
\hline
\end{tabular}




\begin{tabular}{|c|c|c|c|}
\hline AVALIAIFS & $\begin{array}{l}\text { Aplicativo para avaliação institucional, } \\
\text { gera indicadores }\end{array}$ & $\begin{array}{l}\text { Bases de dados do INEP / } \\
\text { MEC }\end{array}$ & $01 / 11 / 2016$ \\
\hline $\begin{array}{l}\text { INFRAERO } \\
\text { VOOS ONLINE }\end{array}$ & $\begin{array}{l}\text { Informações sobre voos e } \\
\text { funcionamento de aeroportos }\end{array}$ & Bases da Infraero & $30 / 03 / 2016$ \\
\hline CÂMBIO LEGAL & $\begin{array}{l}\text { Informações e localização de pontos } \\
\text { de câmbio }\end{array}$ & Bases do banco central & $14 / 02 / 2017$ \\
\hline $\begin{array}{l}\text { INFRAERO } \\
\text { AEROPERTO }\end{array}$ & $\begin{array}{l}\text { Informações de localização de } \\
\text { aeroportos, lojas e os serviços } \\
\text { localizados no terminal }\end{array}$ & $\begin{array}{l}\text { Bases da Infraero e } \\
\text { TripAdvisor }\end{array}$ & $14 / 02 / 2014$ \\
\hline MOBGUIA & $\begin{array}{l}\text { Aplicativo para distribuição de } \\
\text { conteúdo via internet sobre feiras, } \\
\text { eventos e turismo }\end{array}$ & Desconhecido & $16 / 02 / 2016$ \\
\hline CNPJ & Informações sobre CNPJ e CNAE & Bases receita federal & $08 / 10 / 2015$ \\
\hline $\begin{array}{l}\text { ANATEL } \\
\text { SERVIÇO } \\
\text { MÓVEL }\end{array}$ & $\begin{array}{l}\text { Informações para usuários da telefonia } \\
\text { móvel celular sobre qualidade da rede, } \\
\text { localização das estações (antenas) de } \\
\text { cada prestadora e as tecnologias } \\
\text { presentes }\end{array}$ & Bases da Anatel & $18 / 11 / 2016$ \\
\hline $\begin{array}{l}\text { SOFTWARE } \\
\text { PÚBLICO } \\
\text { BRASILEIRO }\end{array}$ & $\begin{array}{l}\text { Informações sobre os softwares } \\
\text { disponíveis no Portal do Software } \\
\text { Público Brasileiro. }\end{array}$ & Portal do Software Público & $09 / 11 / 2015$ \\
\hline E-RECURSOS & $\begin{array}{l}\text { Consulta andamento dos processos } \\
\text { de recursos administrativos de } \\
\text { benefícios, no âmbito da Previdência } \\
\text { Social }\end{array}$ & Bases da Previdência Social & $23 / 04 / 2014$ \\
\hline $\begin{array}{l}\text { RASTROS DA } \\
\text { LEI }\end{array}$ & $\begin{array}{l}\text { Informações sobre acompanhamento } \\
\text { dos projetos de leis federais até sua } \\
\text { ratificação ou arquivação }\end{array}$ & $\begin{array}{l}\text { Site Senado Federal e } \\
\text { Câmara dos Deputados }\end{array}$ & $30 / 03 / 2015$ \\
\hline DETRAN MÓVEL & $\begin{array}{l}\text { Acesso a serviços online oferecidos } \\
\text { pelo Detran-DF. }\end{array}$ & Bases do Detran-DF & $04 / 08 / 2016$ \\
\hline $\begin{array}{l}\text { SIAT MOBILE - } \\
\text { BOMBEIROS ES }\end{array}$ & $\begin{array}{l}\text { Informações sobre estabelecimento } \\
\text { que possuem alvará do Corpo de } \\
\text { Bombeiros do Espírito Santo }\end{array}$ & $\begin{array}{l}\text { Bases do Governo do Espírito } \\
\text { Santo }\end{array}$ & $14 / 10 / 2016$ \\
\hline $\begin{array}{l}\text { DESAPARECIDO } \\
\text { SBRASIL.ORG }\end{array}$ & $\begin{array}{l}\text { Informações sobre pessoas } \\
\text { desaparecidas e encontradas }\end{array}$ & $\begin{array}{l}\text { Portal de desaparecidos do } \\
\text { governo Federal } \\
\text { (http://www.desaparecidos.go } \\
\text { v.br/) }\end{array}$ & $27 / 05 / 2015$ \\
\hline $\begin{array}{l}\text { BOLETIM AO } \\
\text { MAR }\end{array}$ & $\begin{array}{l}\text { Informações sobre o serviço } \\
\text { metereológico marinho }\end{array}$ & \begin{tabular}{|l|} 
Bases da Marinha e de \\
Metereologia
\end{tabular} & $21 / 01 / 2017$ \\
\hline $\begin{array}{l}\text { PORTAL } \\
\text { HIDROLÓGICO } \\
\text { DO NORDESTE }\end{array}$ & $\begin{array}{l}\text { Informações sobre reservatórios } \\
\text { hídricos do Nordeste e Semiárido } \\
\text { Brasileiro }\end{array}$ & $\begin{array}{l}\text { Bases da ANA (Agência } \\
\text { Nacional da Águas) e } \\
\text { FUNCEME (fundação } \\
\text { Cearence de Meteorologia) }\end{array}$ & 09/06/2014 \\
\hline MADEIRA LEGAL & $\begin{array}{l}\text { Informações sobre a rastreabilidade } \\
\text { da madeira e os relatórios de } \\
\text { produção das concessões florestais } \\
\text { federais do Serviço Florestal Brasileiro }\end{array}$ & Desconhecido & $25 / 01 / 2016$ \\
\hline
\end{tabular}

Fonte: Dados da pesquisa.

De maneira geral observou-se que a fonte de dados está diretamente relacionada aos conteúdos produzidos pelo órgão que construiu o aplicativo. Predomina a replicação de serviços habitualmente disponibilizados em portais e poucos aplicativos mesclam com outras fontes do governo. A origem dos dados de alguns aplicativos, como demonstrado no Quadro 3, é desconhecida. Denota-se que o recurso mais utilizado é o serviço de geolocalização disponibilizado, em sua maioria, através de uma Application Programming 
Interface (API). O Google Maps Geolocation API é um exemplo de uma API gratuita utilizada por muitos desenvolvedores de software e governos.

\section{CONSIDERAÇÕES FINAIS}

Pela potencialidade e o dispêndio de tempo na organização para a disponibilização de dados é importante que os DGA sejam reutilizados em novos produtos e serviços inclusive pela própria Administração Pública. $\mathrm{Na}$ pesquisa exploratória no Guia de Aplicativos do Governo Federal identificou-se o acesso e as fontes utilizadas pelos aplicativos desenvolvidos pelos órgãos públicos com relação ao conceito e uso dos DGA.

Em dezembro de 2016 o Guia de Aplicativos do Governo Federal já possuía um total de 129 aplicativos sendo o Android a plataforma dominante. Pela falta de informação relativa à forma de acesso e as fontes de informações utilizadas em cada aplicativo, foi realizada uma análise para identificar esses itens. Como a análise foi baseada no aplicativo e não em seu código fonte, a mesma foi realizada à luz dos conceitos e definições sobre DGA, através das informações disponibilizadas no Guia e através da observação direta ao testar os aplicativos. Não foi possível chegar a uma análise exata dos DGA utilizados nos aplicativos, além disso, alguns DGA são disponibilizados através de portais I serviços, que foram considerados como dados abertos, já que qualquer cidadão poderia realizar uma consulta. Contudo, toda a complexidade envolvida nesta análise, juntamente com as experiências vivenciadas foram muito relevantes para um diagnóstico do uso dos DGA no Brasil.

Um dos objetivos do Guia é disponibilizar aplicativos gratuitos aos cidadãos, porém a forma de acesso pode inibir a acessibilidade e restringir o seu uso. Dos aplicativos disponibilizados no Guia, 46 não foram analisados por não estarem disponíveis na plataforma Android ou por exigir cadastro ou login ou apresentarem erro, ou estarem indisponíveis ou duplicados ou por incompatibilidade com o ambiente de teste. Alguns aplicativos realmente são exclusivos para determinadas categorias como o Sigepe, aplicativo com informações funcionais de servidores ativos, aposentados e pensionistas do Executivo Federal e do Governo do Distrito Federal, contudo alguns aplicativos 
como jogos com temática geral não informam o motivo da exigência de cadastro.

Da amostra analisada, 83 aplicativos, é importante ressaltar que 30, ou seja, $36 \%$ demonstraram utilizar DGA, o que representa um crescimento significativo, já que oficialmente no Portal Brasileiro de Dados Abertos existe o registro de apenas 11 aplicativos, produzidos pela sociedade civil, que utilizam oficialmente DGA, juntamente com o portal de visualização de dados, produzido por organizações públicas.

Da amostra analisada, 63\% dos aplicativos não utilizam DGA ou são jogos off-line que utilizam uma temática específica e não fazem uso dos DGA. Estes dados demonstram que ainda há um potencial inexplorado visto que são disponibilizados, mais de dois mil conjuntos de dados no portal de dados abertos (BRASIL, 2017) e a quantidade de aplicativos que utilizam estes DGA ainda é muito pequena. $O$ incentivo à utilização dos dados tem gerado resultados positivos, o guia destaca os aplicativos que foram desenvolvidos em concursos como o Inovapps 2014 do Ministério das Comunicações. Os concursos, também chamados de hackathon ou maratonas de programação, são iniciativas promovidas pelo governo ou empresas para estimular a exploração de dados abertos e/ou desenvolverem aplicações específicas.

A falta de padronização e os formatos dos dados disponibilizados podem influenciar diretamente no reuso dos dados abertos. Hoje ainda existem conjuntos de dados que são disponibilizados em formato '.pdf', por exemplo, o que dificulta sua reutilização, já que será necessário converter os dados em outros formatos para sua integração. Manter a interoperabilidade dos dados é cada vez mais importante para o ciclo de vida da informação. Abrigar diversos tipos de aplicativos sem conteúdo governamental e sem atualização pode comprometer a finalidade do Guia, assim como jogos com uma temática geral poderiam ser disponibilizados em outras categorias ou canais de comunicação.

Com a análise de todos os aplicativos do Guia é percebida uma evolução no uso dos DGA brasileiros. Mas, em relação ao imenso potencial dos DGA, o Brasil ainda pode ser considerado iniciante no que diz respeito ao uso dos DGA em produtos e serviços de informação principalmente em sua 
reaplicação em inovações. Apesar do Guia disponibilizar aplicativos que não necessariamente utilizam DGA, espera-se que em curto espaço de tempo ele seja orientado pelo conceito da não restrição. A partir do objetivo proposto o resultado da pesquisa conduz a estimular o uso dos DGA, a divulgação das fontes e conjuntos de dados que possam ser reutilizados para 0 desenvolvimento de novos aplicativos, o estabelecimento de interfaces entre diferentes sistemas e a necessária padronização de formatos de arquivos.

\section{REFERÊNCIAS}

AGUNE, R. M.; GREGORIO FILHO, A. S.; BOLLIGER, S. P. Governo aberto SP: disponibilização de bases de dados e informações em formato aberto. In: CONGRESSO CONSAD DE GESTÃO PÚBLICA, 3., 2010. Anais eletrônicos... Brasília, 2010. Disponível em: http://www.prefeitura.sp.gov.br/cidade/secretarias/upload/controladoria_geral/ar quivos/C3_TP_GOVERNO\%20ABERTO\%20SP\%20DISPONIBILIZACAO\%20 DE\%20BASES\%20DE\%20DADOS.pdf. Acesso em: 01 jul. 2018.

ALBANO, C. S.; ARAUJO, M. H. de. Inteligência competitiva e monitoramento ambiental utilizando informações disponíveis na internet: a viabilidade de utilizar dados governamentais abertos. In: ENCONTRO DE ADMINISTRAÇÃO DA INFORMAÇÃO (EnADI), 4., 2013. Anais... Bento Gonçalves: EnADI, 2013.

ALEXOPOULOS, C.; ZUIDERWIJK, A.; CHARAPABIDIS, Y.; LOUKIS, E.; JANSSEN, M. Designing a secondgenerationof open data platforms: Integrating open data and social media. In: JANSSEN, M.; SCHOLL, H. J.; WIMMER, M. A.; BANNISTER, F. (Org.). Electronic Government. EGOV 2014. Lecture Notes in Computer Science. Springer, Berlin, 2014. p. 230-241. Disponível em: https://link.springer.com/chapter/10.1007/978-3-662-44426-9_19. Acesso em: 01 jul. 2018.

BARBALHO, F. A. Emergência de um campo de ação estratégica: o caso de política pública sobre dados abertos. 2014. $254 \mathrm{f}$. Tese (Doutorado em Administração) - Universidade de Brasília, Brasília, 2014. Disponível em: http://repositorio.unb.br/bitstream/10482/18041/1/2014_FernandoAlmeidaBarba Iho.pdf. Acesso em: 01 jul. 2018.

BRASIL. Lei $n^{\circ} 12.527$ de 18 de novembro de 2011. Regula o acesso a informações previsto no inciso XXXIII do art. 5o, no inciso II do $\S 3$ o do art. 37 e no § 2 o do art. 216 da Constituição Federal; altera a Lei no 8.112 , de 11 de dezembro de 1990; revoga a Lei no 11.111, de 5 de maio de 2005, e dispositivos da Lei no 8.159, de 8 de janeiro de 1991; e dá outras providências. Diário Oficial da União, Seção 1, 18 nov. 2011, p. 1. 
BRASIL. Guia de Aplicativos Governo Federal. 2016. Disponível em: http://www.aplicativos.gov.br. Acesso em: 10 jan. 2017.

BRASIL. Portal Brasileiro de Dados Abertos. Brasília: 2017. Disponível em: http://dados.gov.br. Acesso em: 01 jul. 2018.

DUTRA, C. C.; LOPES, K. M. G. Dados abertos: uma forma inovadora de transparência. In: CONGRESSO CONSAD DE GESTÃO PÚBLICA, 6., 2013. Disponível em:

http://banco.consad.org.br/bitstream/123456789/915/1/C6_TP_DADOS\%20AB ERTOS\%20UMA\%20FORMA.pdf. Acesso em: 01 jul. $201 \overline{8}$.

GOOGLE. Google Play Store. 2017. Disponível em:

https://play.google.com/store. Acesso em: 20 jan. 2018.

GRAY, J. A data revolution for whom? Open Democracv UK. 2015. Disponível em: https://www.opendemocracy.net/ourkingdom/jonathangrey/data-revolution-for-whom. Acesso em: 20 jan. 2018.

IDC. Smartphone OS Market Share, 2016 Q3. 2016. Disponível em: http://www.idc.com/promo/smartphone-market-share/os. Acesso em: 01 jul. 2018.

IDC BRASIL. Estudo da IDC Brasil aponta vendas de 10.3 milhões de celulares no primeiro trimestre. 2016. Disponível em:

http://br.idclatin.com/releases/news.aspx?id=2044. Acesso em: 01 jul. 2018.

ISOTANI, S.; BITTENCOURT, I. I. Dados Abertos Conectados: Em busca da Web do Conhecimento. São Paulo: Novatec, 2015.

KALAMPOKIS, E.; TAMBOURIS, E.; TARABANIS, K. A classification scheme for open government data: towards linking decentralised data. International Journal of Web Engineering and Technology, v. 6, n. 3, p. 266-285, 2011. Disponível em:

http://citeseerx.ist.psu.edu/viewdoc/download?doi=10.1.1.703.4909\&rep=rep1\&t ype=pdf. Acesso em: 01 jul. 2018.

MCKINSEY GLOBAL INSTITUTE. Open data: Unlocking innovation and performance with liquid information. McKinsey \& Company, 2013. Disponível em: https://www.mckinsey.com/business-functions/digital-mckinsey/ourinsights/open-data-unlocking-innovation-and-performance-with-liquidinformation. Acesso em: 01 jul. 2018.

MOREIRA, D. L. de J. Panorama sobre a utilização de dados governamentais abertos no Brasil: um estudo a partir dos aplicativos desenvolvidos. 2015. 158 f. Dissertação (Mestrado em Ciência da Informação) - Universidade Federal do Rio de Janeiro, Rio de Janeiro, 2015. Disponível em: http://ridi.ibict.br/bitstream/123456789/792/1/Mestrado_Diogo_Luiz_Jesus_Mor eira_2015.pdf. Acesso em: 01 jul. 2018. 
OPEN GOV DATA. Eight principles of open government data. Califórnia, 2007. Disponível em: https://public.resource.org/8_principles.html. Acesso em: 01 jul. 2018.

SOLAR, M.; DANIELS, F.; LÓPEZ, R.; MEIJUEIRO, L. A Model to Guide the Open Government Data Implementation in Public Agencies. Journal Of Universal Computer Science, v. 20, n. 11, p. 1564-1582, 2014. Disponível em:

https://pdfs.semanticscholar.org/361a/aec3cf949217469abc9a4795be84abf56a 08.pdf. Acesso em: 01 jul. 2018.

VAN DEN BROEK, T.; RIJKEN, M.; VAN OORT, S. Towards Open Development Data: A review of open development data from a NGO perspective. Holanda, jul. 2012. Disponível em: https://repository.tudelft.nl/view/tno/uuid:c1ef3a5a-155d-4139-bb47360a401ca339/. Acesso em: 01 jul. 2018.

W3C. Dados Abertos Governamentais. World Wide Web Consortium Escritório Brasil. 2016. Disponível em: http://www.w3c.br/divulgacao/pdf/dadosabertos-governamentais.pdf. Acesso em: 01 jul. 2018.

YANNOUKAKOU, A.; ARAKA, I. Access to government information: Right to information and open government data synergy. Procedia-Social and Behavioral Sciences, v. 147, p. 332-340, 2014. Disponível em: https://www.sciencedirect.com/science/article/pii/S187704281404018X. Acesso em: 01 jul. 2018.

\title{
OPEN GOVERNMENT DATA IN BRASILIAN APPLICATIONS
}

\begin{abstract}
Introduction: Public data provided by governments through the internet are identified as Open Government Data (OGD). By making the data available, the research seeks to evaluate if they are reused by society and through the applications developed by the Brazilian government in products and information services. Objective: To identify the applications made available by the Brazilian government and their degree of reuse based on the use of the OGD. Methodology: Through an exploratory research with a qualitative and quantitative approach, the applications available in the Federal Government's Application Guide were analyzed and access criteria, data sources and especially OGD were analyzed. Results: We identified 30 applications that use OGD, an expressive number of reuse when compared to the number of official applications produced with OGD of the Brazilian government. But there are still a lot of games and applications that do not use OGD. Conclusions: There is an evolution in the use of Brazilian OGD, but in relation to the immense potential of the OGD, Brazil still walks inexpressively with regard to the use of OGD in information products and services.
\end{abstract}

Descriptors: Open government data. Government information. Information policies. 


\title{
DATOS DE GOBIERNO ABIERTO EM LAS APLICACIONES BRASILEÑAS
}

\begin{abstract}
RESUMEN
Introducción: Los datos públicos proporcionados por los gobiernos a través de Internet se identifican los Datos de Gobierno Abierto (DGA). Al hacer que los datos disponibles, la investigación busca evaluar si son reutilizados por la sociedad y por medio de las aplicaciones desarrolladas por el gobierno brasileño en los productos y servicios de información. Objetivo: Identificar las aplicaciones proporcionadas por el gobierno brasileño y su grado de reutilización basado en el uso de la DGA. Metodología: A través de una investigación exploratoria con enfoque cualitativo y cuantitativo analiza las aplicaciones disponibles en la Guía de Aplicación del Gobierno Federal y evaluado los criterios de acceso, fuentes de datos y utiliza principalmente DGA. Resultados: Se identificaron 30 aplicaciones que utilizan DGA, un número significativo de reutilización compararon la cantidad de aplicaciones oficiales producidos con DGA el gobierno brasileño. Pero todavía hay una cantidad significativa de juegos y aplicaciones que no utilizan DGA. Conclusiones: Hay una evolución en el uso de la DGA de Brasil, sino en relación con el inmenso potencial de la DGA, Brasil todavía camina sin expresión en relación con el uso de DGA en los productos y servicios de información.
\end{abstract}

Descriptores: Datos de gobierno abierto. Información gubernamental. Política de información. 